\title{
The Use of 'Live Chemical Imaging' to Enhance and Increase Productivity in SEM/EDS Investigation of Pharmaceutical Samples, While Still Conforming to 21 CFR Part 11 Regulations
}

\author{
Anthony Hyde ${ }^{1}$, Simon Burgess ${ }^{1}$, and Jenny Goulden ${ }^{1}$ \\ 1. Oxford Instruments, High Wycombe, United Kingdom
}

This paper details the application of 'Live Chemical Imaging' (LCI) to enhance and increase productivity when using energy dispersive spectroscopy (EDS) in the scanning electron microscope (SEM). The workflow for investigating pharmaceutical samples is reviewed with respect to helping users conform to regulations contained within 21 CFR Part 11.

The US Food and Drug Administration (FDA) regulation 21 CFR part 11 [1] covers the use of electronic records and electronic signatures in the regulated pharmaceutical, biotechnology and medical device industries. It is intended to ensure that electronic records are trustworthy with a full audit trail. However, if not implemented carefully, the requirements of this regulation can make the routine workflow associated with EDS analysis in the SEM lengthy and inefficient. Routine analysis workflows for analysis in the SEM typically involve a quick 'preliminary' sample examination to obtain a broad sample overview before commencing more detailed analysis. This preliminary examination involves scanning regions of the sample, to identify areas of interest, and will include collecting analytical data; images, spectra and Xray maps to locate these specific features of interest. In many cases this data is not relevant to the final analysis and in a routine workflow would be deleted.

However, when working within the requirements of 21 CFR part 11, the deletion or modification of data is either not permitted or must be accompanied by digital signatures and a documented explanation. This requirement can make the sample examination step an inefficient part of a typical workflow.

The term 'Live Chemical Imaging (LCI)' refers to the live, continuous and simultaneous acquisition of an electron image, X-ray maps and spectrum (with automatic peak identification) whilst scanning the sample. This technique has two advantages. Firstly, it enables the user to carry on with their existing pre-analysis without worrying about deleting data or adding digital signatures, because no data is saved. Secondly, the user can investigate their sample more quickly, easily and with greater detail than previously possible. To validate this approach two routine workflows were investigated in this study:

Pharmaceutical Quality Control - Contamination: A metered dose inhaler (MDI) is a device that delivers a specific amount of medication to the lungs, in the form of a short actuation of aerosolized medicine (used to treat ailments such as asthma). It is vital these actuations are free from contamination. LCI offers a quick method for reviewing quality medication, easily identifying harmful contaminants.

Identifying Counterfeit Products: The production and sale of counterfeit pharmaceutical products is a global problem. Estimates of the loss to the pharmaceutical industry range from $\$ 160$ to $\$ 200$ billion a year. In addition there is the risk of potentially harmful products making their way into consumer use. Globally, government bodies use a host of analytical techniques to identify counterfeit products. In this example 3 pain relief tablets were prepared (1) Leading brand (2) Suspected counterfeit claiming to be leading brand (3) Generic claiming to have the same ingredients as the leading brand. Here LCI enabled 
the differences between the three samples to be clearly recognized before the full analysis was undertaken. Analysis shows that Sample 1 and 2 were similar in composition but different in structure and therefore sample 2 was confirmed as a counterfeit product. Sample $C$ was very different in both structure and composition, and further analysis is recommended to determine any health risks from ingesting such a product. X-ray maps and spectra from the three samples are shown in Figures 1, 2 and 3 below. In each case LCI was applied to identify the region prior to data collection. In addition, videos of LCI being used to investigate the three samples can be viewed via QR code, Figure 4.

\section{References:}

[1] FDA (https://www.fda.gov/downloads/regulatoryinformation/guidances/ucm125125.pdf)

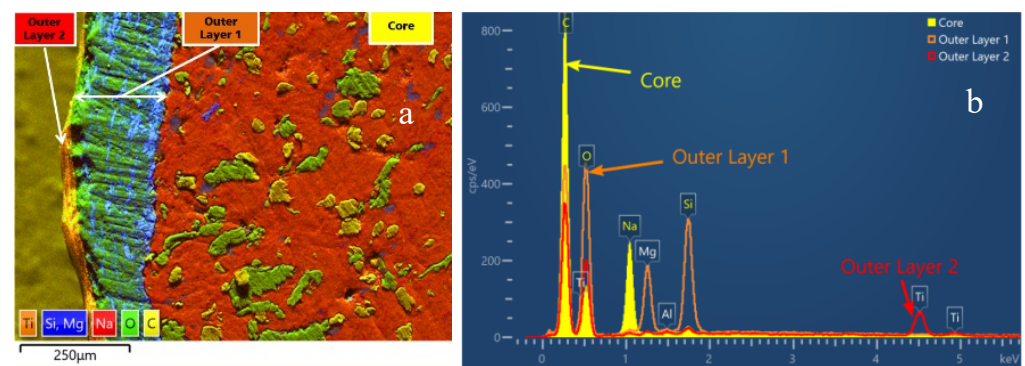

Figure 1a and 1b. Sample 1. Layered Image, Comparison Spectra (from conventional EDS analysis).
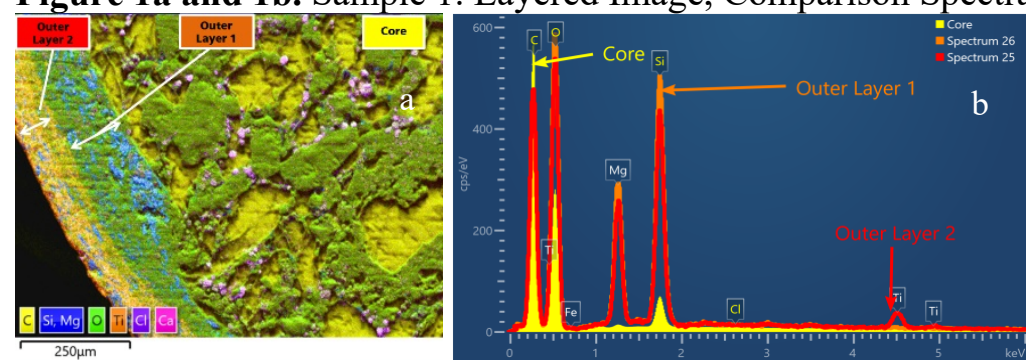

Figure 2a/b. Sample 2 Layered Image, Comparison Spectra (from conventional EDS analysis).
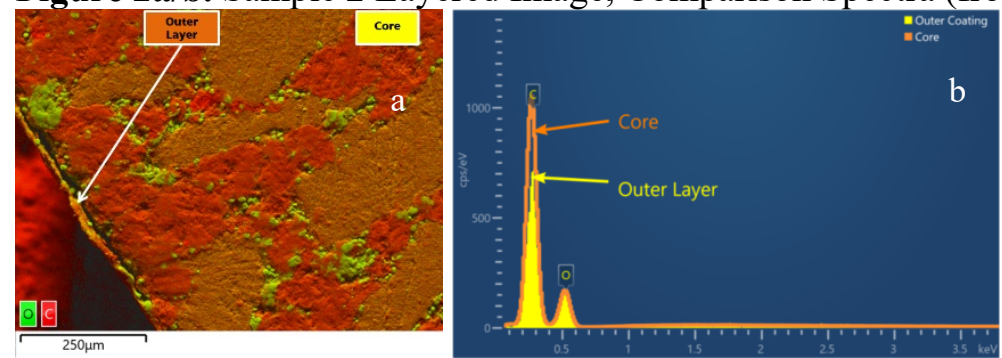

Figure 3a and 3b. Sample 3 Layered Image, Comparison Spectra (from conventional EDS analysis).

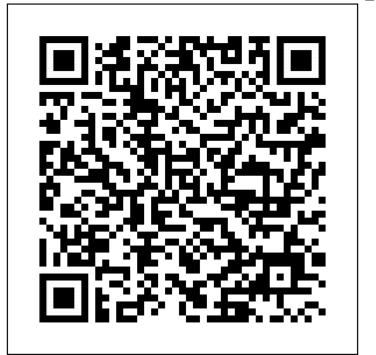

Figure 4. Smart Phone machine readable QR code, linking to videos of LCI investigation of Samples 1-3. 